\title{
More blastocysts are produced from fewer oocytes in ICSI compared to IVF - results from a sibling oocytes study and definition of a new key performance indicator
}

\author{
Sandrine Chamayou ${ }^{1 *}\left(\mathbb{D}\right.$, Carmen Ragolia $^{1}$, Carmelita Alecci', Giorgia Storaci ${ }^{1}$, Simona Romano', \\ Roberta Sapienza' ${ }^{1}$ Elena Maglia', Annalisa Liprino ${ }^{1}$, Clementina Cardea' ${ }^{1}$ Michele Fichera² and \\ Antonino Guglielmino'
}

\begin{abstract}
Background: Which fertilization method, between ICSI and IVF in split insemination treatments, has the highest clinical efficiency in producing clinically usable blastocyst?

Methods: 211 infertile couples underwent split insemination treatments for a non-severe male factor. 1300 metaphase II (MII) oocytes were inseminated by conventional IVF and 1302 MII oocytes were micro-injected with the same partner's semen. Embryo development until blastocyst stage on day $V$ and clinical outcomes were valuated trough conventional key performance indicators (KPI), and new KPIs such as blastocyst rate per used MII oocytes and the number of MII Oocytes to produce one clinically usable blastocyst from ICSI and IVF procedures.
\end{abstract}

Results: The results were globally analyzed and according to ovarian stimulation protocol, infertility indication, and female age. The conventional KPI were online with the expected values from consensus references. From global results, $2.3 \mathrm{MII}$ oocyte was needed to produce one clinically usable blastocyst after ICSI compared to $2.9 \mathrm{MII}$ oocytes in IVF. On the same way, more blastocysts for clinical use were produced from fewer MII oocytes in ICSI compared to IVF in all sub-groups.

Conclusions: In split insemination treatments, the yield of clinically usable blastocysts was always superior in ICSI compared to IVF. The new KPI "number of needed oocytes to produce one clinically usable embryo" tests the clinical efficiency of the IVF laboratory.

Keywords: Blastocyst, ICSI, IVF, Key performance indicator, Sibling oocyte, Split insemination

\section{Key Message}

In split insemination study treatments, the yield of clinically usable blastocysts is superior in ICSI compared to IVF. The new KPI "number of needed oocytes to produce

*Correspondence: s.chamayou@yahoo.fr

1 Unità di Medicina della Riproduzione - Centro HERA, via barriera del bosco, n. 51/53, Sant'Agata Li Battiati, Catania, Italy

Full list of author information is available at the end of the article one clinically usable embryo" tests the clinical efficiency of the IVF laboratory.

\section{Introduction}

The two main in vitro fertilization techniques are conventional In Vitro Fertilization (IVF) [1] and IntraCytoplasmic Sperm Injection (ICSI) [2, 3]. Conventional IVF solves gynaecological infertility such as tubal factor, endometriosis, anovulation, unexplained infertility, and original author(s) and the source, provide a link to the Creative Commons licence, and indicate if changes were made. The images or other third party material in this article are included in the article's Creative Commons licence, unless indicated otherwise in a credit line to the material. If material is not included in the article's Creative Commons licence and your intended use is not permitted by statutory regulation or exceeds the permitted use, you will need to obtain permission directly from the copyright holder. To view a copy of this licence, visit http://creativecommons.org/licenses/by/4.0/. The Creative Commons Public Domain Dedication waiver (http://creativeco mmons.org/publicdomain/zero/1.0/) applies to the data made available in this article, unless otherwise stated in a credit line to the data. 
moderate male factor [4]. ICSI solves severe male factor [2], unexplained infertility with previous fertilization failure [5], is commonly applied in case of reduced oocyte yield [6] and when thawed oocytes are used [7]. Consequently, and worldwide, we observe an increasing use of ICSI over IVF that reached $71.3 \%$ of fresh in vitro treatments [8]. In Italy, conventional IVF with fresh and non-donated gametes was applied in only $15.2 \%$ of the assisted fertilization treatments in 2019 [9].

Sincethe early 2000' and in different IVF units, the split insemination strategy hasbeen applied to compare IVF and ICSI results from oocytes produced from thesame ovarian stimulation (sibling oocytes) $[10,11]$. Throughthis strategy, it is possible to verify if gametes are competent to fertilize bythemselves in IVF or if they need to be assisted by ICSI. In the meantime, theembryo transfer should be ensured from embryos produced in ICSI.

Different groups have compared IVF and ICSI results and efficacy on sibling oocytes in different groups of patients. The Key Performance Indicators (KPIs) applied to compare the two fertilization methods were fertilization and cleavage rates, embryo quality, the percentage of transferred and frozen embryos on zygote number, and clinical outcome measurements such as implantation and clinical pregnancy rates. Different studies found the two fertilization methods comparable and suggest applying IVF when possible [12-14] even when the fertilization rate was higher in ICSI [11, 15-17]. In all cases, the authors tested the goodness of IVF and ICSI procedures in their lab from a technical perspective. The expected values of the laboratory KPIs are currently reported in consensus references [18-20]. On the other side, the real efficiency of each fertilization procedure in producing an embryo for clinical use and a live baby born for the studied populations was not estimated in the above studies. Consequently, and based on systematic reviews and meta-analyses of previously published data, the (non-) advantage of ICSI over IVF for non-severe male factor can still be considered an open question [21,22].

To evaluate the real reproductive prospective of ICSI and IVF, we need to compare the two fertilization methods from a clinical point of view using laboratory data in split insemination treatments. New KPIs such as the "number of metaphase II (MII) oocytes needed to produce one clinically usable blastocyst" will help in understanding which of the two methods produces the highest number of clinically usable blastocysts for fresh and postponed clinical use.

In our center and since 1997, split insemination treatments are applied in accordance with the physician and the patients based on their previous infertility history and the male factor status. Since 2011, embryo culture is performed under time-lapse monitoring in our IVF lab [23].
Consequently, data from split insemination treatments are easily accessible and can be retrospectively analyzed.

In the present study, we compared retrospective in vitro results and clinical outcomes after blastocyst transfer on day $\mathrm{V}$ of split insemination treatments (conventional IVF and ICSI). The results were analyzed in their globality and according to ovarian stimulation protocol, infertility indication, and female patient age group. To perform these analyses, conventional and new KPIs were used to test each fertilization method's efficiency in producing the highest number of blastocysts for clinical use.

\section{Materials and methods}

All procedures were approved by our Institutional Review Board. All participants gave written consent on all aspects of the treatment after having been informed.

\section{Patients and ovarian stimulation}

Between March 2011 and March 2020, 334 couples underwent split insemination treatments. For the present study, only couples in which embryo-culture was continued until day $\mathrm{V}$ were selected. The fate of embryo to transfer or freezing was based on embryo morphology and morphokinetic only, and not on fertilization method. Furthermore, we included only cases in which the final fate of each blastocyst produced from ICSI or IVF was determined: embryo-transfers with only ICSI embryos; embryo-transfers with only IVF embryos; and embryotransfers with embryos from both ICSI and IVF and with the same fate (none implanted or both implanted). The embryo-transfers with embryos from both ICSI and IVF in which only one embryo implanted were excluded from the present study. Consequently, 211 cases were selected.

The infertility indications were anovulation (24 couples), endometriosis (14 couples), male factor (20 couples), polycystic ovarian syndrome (5 couples), tubal factor (57 couples), and unexplained infertility (91 couples). Female patients were aged between 19.8 and 44.3 years (mean age: 33.9 ), and basal FSH on Day III was between 2.7 and $12.8 \mathrm{IU} / \mathrm{l}$ (mean $4.5 \mathrm{IU} / \mathrm{l}$-Immulite 2000, Siemens-Germany). Karyotype was normal for all patients. To perform split insemination treatment, the semen parameters needed to be suitable to perform conventional IVF. Patients with severe oligotheratoastenozoospermia were excluded.

Two different protocols of ovarian stimulations were carried out. In the long protocol, agonist (gonadotrophin-releasing hormone-GnRHa, Suprefact: Hoechst Marion Roussel Deutschland GmbH, Frankfurt, Germany) started in the luteal phase and was followed by delay administration of recombinant FSH (Gonal-F: Merck-Serono, London, UK or Puregon, MSD, Franklin 
Lakes, USA) and LH (Luveris: Merck-Serono) after down-regulation. In the short protocol with an antagonist, ovarian stimulation was performed by administering recombinant FSH and LH from cycle Day III. When the leading follicle reached $14 \mathrm{~mm}$ in diameter, the antagonist was daily added with a dosage of $0.25 \mathrm{mg} /$ day (Cetrotide: Merck-Serono, London, UK) until triggering. In both protocols, initial doses were 150-300 IU/day for FSH and 75-150 IU/day for LH. Triggering was done with HCG 10,000 IU (Gonasi: AMSA, Italy). Vaginal ultrasound-guided aspiration of oocyte - cumulus complexes was performed $35 \mathrm{~h}$ after human chorionic gonadotrophin administration.

\section{Split insemination (ICSI and IVF) and embryo culture}

After oocyte retrieval, the cumulus-oocyte complexes $(\mathrm{COC})$ were randomly divided into two groups. Gamete preparations were previously described [2, 3, 24]. In the first group and after cumulus cells elimination, MII oocytes were micro-injected with the partner's freshly ejaculated spermatozoa three hours after oocyte retrieval, as previously described [24]. In the meantime, and in IVF, each COC was inseminated with 25.000 motile spermatozoa from the same partner's sperm sample for $3 \mathrm{~h}$. After $3 \mathrm{~h}$, cumulus cells were gently removed, and the meiotic state of inseminated oocytes were evaluated with the observation of the first polar body. All the key performance indicators based on the number of MII oocytes in IVF were calculated on the number of MII oocytes at that time.

After micro-injection in ICSI and incubation with partner's sperm in IVF, the cell in vitro culture was placed in 25 ul of continuous, single culture complete medium with human serum albumin (Irvine Scientific, Santa Ana, USA) under mineral oil and in automated incubators with $5 \% \mathrm{CO} 2,5 \% \mathrm{O} 2$ at $37{ }^{\circ} \mathrm{C}$, fitted with time-lapse imaging acquisition (Embryoscope, Unisense, AarhusDenmark). The entire embryo development has been followed and analyzed. During incubation in the Embryoscope, seven plane focal images were generated each $20 \mathrm{~min}$ and recorded.

In all the present cases, the number of available embryos at cleavage stage (day II-III) was superior to the number of embryos decided to be transferred, and the morphokinetic parameters matched with the predictive values to reach blastocyst stage previously established in our lab conditions [23]. Consequently, and in accordance with the patients' and physicians' decisions, embryo culture was continued until blastocyst stage for embryo transfer with/without surplus blastocyst vitrification.

After embryo transfer on day V, clinical pregnancy was ascertained by observation of fetal heartbeat in the successive weeks. Pregnancies were followed until birth.

\section{Blastocyst vitrification}

The surplus blastocysts were vitrified according to the vitrification protocol previously described $[24,25]$.

\section{Key performance indicators}

The conventional KPIs in assisted reproductive techniques were applied: fertilization rate, cleavage and blastocyst rates calculated per zygote, and implantation rate calculated per transferred embryos $[18,26]$.

Live birth rate was calculated per transferred embryos.

The new KPIs were the blastocyst rate per (microinjected or inseminated) MII oocyte, and the number of MII oocytes needed to produce one clinically usable blastocyst on day V (to transfer or to freeze).

All rates were compared between IVF and ICSI on sibling gametes.

\section{Statistical analysis}

As the size of studied samples was superior to 25 , the statistical significance of rates was tested by $\mathrm{z}$ test at different levels of significance $(p<0.05,0.01,0.001)$. The normal distribution of data was assumed. The null hypothesis was no difference between the checked rates.

\section{Results}

In vitro results and clinical outcomes were analyzed in their totality and according to ovarian stimulation protocol, infertility indication, and female age.

\section{Global results \\ In vitro results}

A total of $2602 \mathrm{COC}$ were retrieved (mean of $12.3 \mathrm{COC}$ per OPU) from which 1302 COC were used for ICSI and 1300 for IVF. After decumulation, it resulted that $900 \mathrm{MII}$ oocytes were inseminated by conventional IVF (mean number: 4.3) and 904 MII oocytes were micro-injected by ICSI (mean number: 4.3). The fertilization rate was statistically superior in ICSI $(729 / 904,80.6 \%)$ compared to IVF $(596 / 900,66.2 \% ; p<0.001)$. There was no statistical difference in the cleavage rate $(709 / 729,97.3 \%$ in ICSI and $573 / 596,96.1 \%$ in IVF; $p>0.05)$ and blastocyst rate per zygote on day V (399/729, 54.7\% in ICSI and 309/596, $51.8 \%$ in IVF; $p>0.05)$ between the two fertilization techniques.

According to the new KPIs, the blastocyst rate calculated on MII oocyte was statistically superior in ICSI (399/904, 44.1\%) compared to IVF (309/900, 34.3\%, $p>0.001)$. Consequently, the number of oocytes needed 
to produce one clinically usable blastocyst was a mean of 2.3 after ICSI and 2.9 after IVF.

\section{Clinical outcomes}

Of the 188 ICSI-blastocysts transferred on Day V, 80 implanted (42.6\%) leading to 72 live births (38.3\%). Of the 178 IVF-blastocysts transferred on day V, 69 implanted $(35.4 \%, p>0.05)$ leading to 63 live births $(35.4 \% ; p>0.05)$ (Table 1$)$.

\section{According to ovarian stimulation protocol, infertility indication and female patient age}

The in vitro results and clinical outcomes after ICSI and IVF on sibling oocytes were divided in sub-groups and analyzed according to ovarian stimulation protocol in 122 couples (mean female age: 34.3 ) after ovarian stimulation with long protocol and 89 couples (mean female age: 33.6) after short protocol; according to infertility indication in 91 couples with unexplained infertility (mean female age: 34.9), 20 couples with male infertility (mean female age: 31.6 ) and 100 couples with female infertility (anovulation, endometriosis, polycystic ovarian syndrome, tubal factor) (mean female age: 33.5 ) and according to female patient age (159 female patients aged between 19 and 37 years old, and 52 aged between 38 and 45 years old) (see Table 2).

Table 1 Comparison of in vitro results after blastocyst transfers after ICSI and IVF on sibling oocytes in split insemination cycles

\begin{tabular}{lllll}
\hline & ICSI & IVF & $\boldsymbol{p}$ & TOTAL \\
\hline n. cycles & 211 & & & $\mathbf{2 1 1}$ \\
n. COCs & 1302 & 1300 & & 2602 \\
n. MII micro-injected or inseminated & 904 & 900 & & 1804 \\
Oocytes & & & & \\
n. zygotes & 729 & 596 & & 1325 \\
fertilization rate & $\mathbf{8 0 . 6}$ & $\mathbf{6 6 . 2}$ & $<\mathbf{0 . 0 0 1}$ & $\mathbf{7 3 . 4}$ \\
n. cleaved embryos & 709 & 573 & & 1282 \\
cleavage rate & $\mathbf{9 7 . 3}$ & $\mathbf{9 6 . 1}$ & NS & $\mathbf{9 6 . 8}$ \\
n. blastocysts & 399 & 309 & & 708 \\
blastocyst rate/ zygote & $\mathbf{5 4 . 7}$ & $\mathbf{5 1 . 8}$ & NS & $\mathbf{5 3 . 4}$ \\
blastocyst rate/ MII oocyte & $\mathbf{4 4 . 1}$ & $\mathbf{3 4 . 3}$ & $<\mathbf{0 . 0 0 1}$ & $\mathbf{3 9 . 2}$ \\
n. MIl oocytes per usable blastocyst & $\mathbf{2 . 3}$ & $\mathbf{2 . 9}$ & $<\mathbf{0 . 0 0 1}$ & $\mathbf{2 . 5}$ \\
n. frozen blastocysts & 211 & 131 & & 342 \\
n. transferred blastocysts & 188 & 178 & & 366 \\
n. implanted blastocysts & 80 & 69 & & 149 \\
implantation rate & $\mathbf{4 2 . 6}$ & $\mathbf{3 8 . 8}$ & NS & $\mathbf{4 0 . 7}$ \\
n. live births & 72 & 63 & & 135 \\
live birth rate & $\mathbf{3 8 . 3}$ & $\mathbf{3 5 . 4}$ & NS & $\mathbf{3 6 . 9}$ \\
\hline
\end{tabular}

\section{In vitro results}

On a global view, the comparability of ICSI and IVF in vitro results were similar through the analyses of ovarian stimulation protocol, infertility indication and female patient age group.

Fertilization rate was always significantly superior in ICSI compared to IVF in all sub-groups. Cleavage rate was equivalent after ICSI and IVF, except for long protocol and female indication sub-groups where it resulted significantly superior in ICSI $(p<0.01)$. Blastocyst rates calculated on zygote were equivalent in both techniques, except for long protocol sub-group where it was significantly superior in ICSI $(p<0.01)$.

According to the new KPIs, blastocyst rate per MII oocyte was superior in ICSI for all groups, reaching statistical significance in long and short protocols, unexplained infertility, female infertility and 19-37 years old groups. Consequently, the micro-injected or inseminated MII oocytes needed to obtain a viable blastocyst on day V, was always inferior in ICSI compared to IVF. It varied from 1.8 in ICSI after long ovarian protocol to 3.0 in IVF for moderate male factor.

All data are resumed in Table 3.

\section{Clinical outcomes}

As for in vitro results, clinical outcomes followed the comparability of ICSI, and IVF in vitro results were similar through the analyses of ovarian stimulation protocol, infertility and indication and female age. In all sub-groups, the implantation rate and live birth rate calculated per transferred embryo were comparable.

\section{Discussion}

In the present study, we compared in vitro results and clinical outcomes in treatments in which half of the oocytes from the same ovarian stimulation were inseminated in conventional IVF, and half of the oocytes were micro-injected with the same partner's sperm in ICSI. The effects of fertilization methods on fertilization rate, embryo development, and competence to lead a live birth were studied.

The results were analyzed on their globality and successively according to the ovarian stimulation protocol, infertility indication, and female age. In the female age sub-group, the division was made between 37 and 38 years old because clinical outcomes and embryo implantation decrease drastically from 38 years old due to increasing embryo aneuploidy [27]. As a mandatory condition for the present study, the number of MII oocytes used for IVF and ICSI was equal and the fate of each transferred blastocyst was known. 


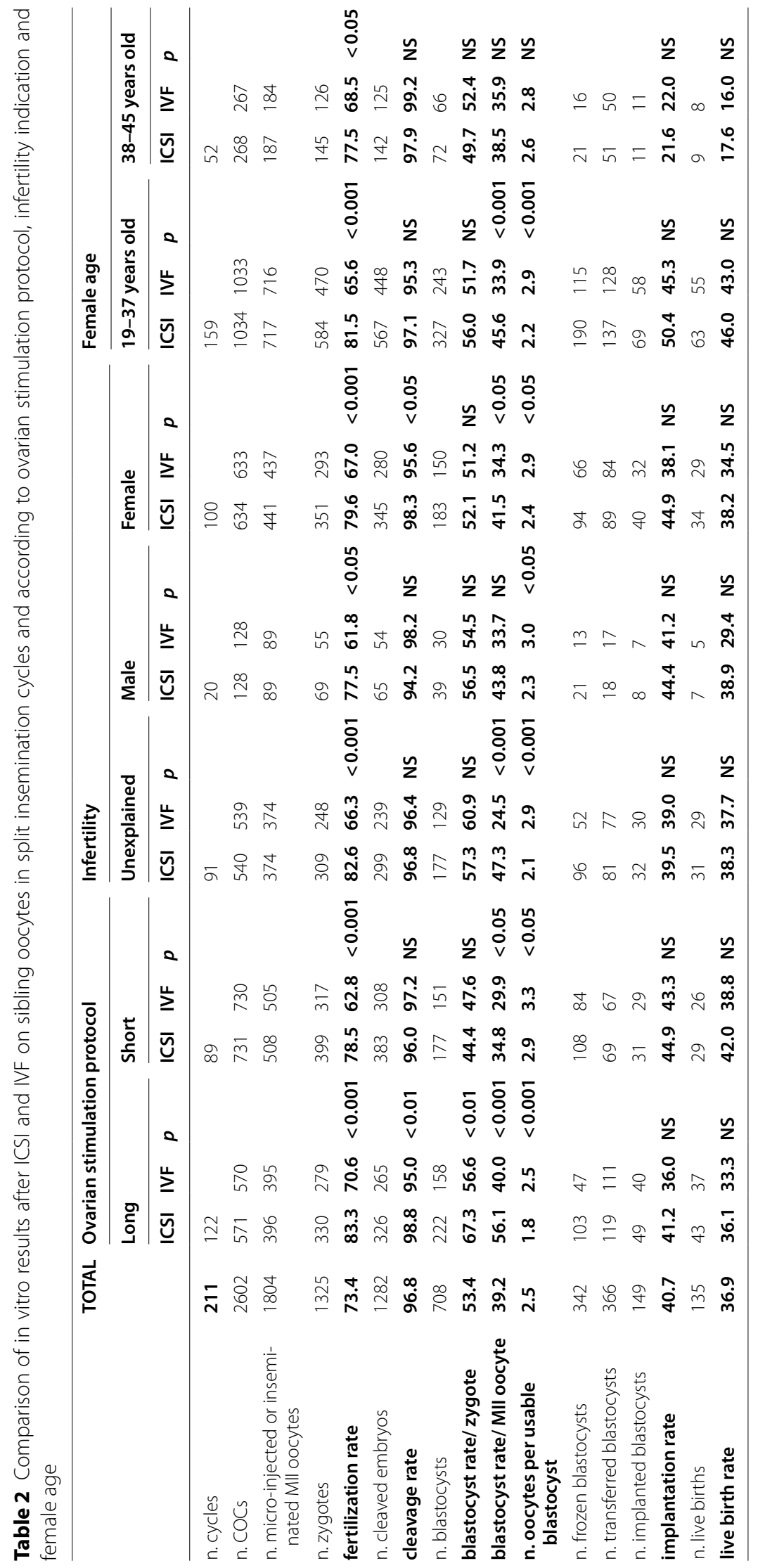


Table 3 Number of MII oocyte per fertilization method to produce one clinically usable embryo

\begin{tabular}{lllll}
\hline n. MII oocytes per usable embryo & \multicolumn{5}{l}{ Transfer at blastocyst stage } \\
\cline { 2 - 5 } & $\mathbf{N}$ & ICSI & IVF & $\boldsymbol{p}$ \\
\hline TOTAL & 211 & 2.3 & 2.9 & $<0.001$ \\
OVARIAN STIMULATION PROTOCOL & & & & \\
$\quad$ Long protocol & 122 & 1.8 & 2.5 & $<0.001$ \\
$\quad$ Short protocol & 89 & 2.9 & 3.3 & $<0.05$ \\
INFERTILITY INDICATION & & & & \\
$\quad$ Unexplained infertility & 91 & 2.1 & 2.9 & $<0.001$ \\
$\quad$ Male infertility & 20 & 2.3 & 3.0 & $<0.05$ \\
$\quad$ Female infertility & 100 & 2.4 & 2.9 & $<0.05$ \\
FEMALE PATIENT AGE & & & & \\
$\quad$ 19-37 years old & 159 & 2.2 & 2.9 & $<0.001$ \\
$\quad$ 38-45 years old & 52 & 2.6 & 2.8 & NS \\
\hline
\end{tabular}

To compare the results, conventional KPIs were applied such as fertilization rate, cleavage and blastocyst rates calculated on the number of zygotes, implantation rate and live birth rate $[18,26]$, and new KPIs were proposed. Analysis by conventional KPI from global data and data from sub-groups (ovarian stimulation protocol, infertility indication and female age), fertilization always resulted statistically superior in ICSI compared to IVF while cleavage rate, known implantation rate, and known live birth rates were comparable in ICSI and IVF. Blastocyst rate calculated on zygote was comparable in ICSI and IVF with statistical significance in the long ovarian stimulation protocol subgroup. All these KPIs are typically used to check IVF laboratory performance [18], even if implantation and live birth rates also depend on clinical procedures such as embryo transfer and uterine receptivity. The present conventional KPI values in ICSI and IVF were concordant with the expected values [18].

In concordance with our results analyzed in their globality and by sub-groups, previous authors applying split insemination found significant higher fertilization rate in ICSI compared to IVF $[11,15-17,28]$, comparable cleavage rate $[10-12,14,15,20]$, no statistical difference in blastocyst rate $[11,13]$, comparable implantation $[10,12]$ and live birth rates $[13,16]$.

Nevertheless, after applying conventional KPIs to test our data, we did not feel that we were testing the true efficiency of each fertilization method from the global data and in each sub-group. Consequently, it was decided to test other KPIs (blastocyst rate calculated on MII oocyte, the number of oocytes needed to produce one embryo for clinical use) that could be more informative on the true potential on ICSI and IVF in obtaining a live birth.
Which fertilization method of ICSI and IVF leads to the highest number of embryos for clinical use and for which group of patients? While the previous conventional KPI tested laboratory performance, the new KPIs tested the technique and in vitro procedures on a clinical and patient group perspective inside the lab. From our data and as a consequence of the highest fertilization rate in ICSI and the comparability of cleavage rate between ICSI and IVF, the blastocyst rate calculated on used oocytes resulted superior in ICSI compared to IVF. On the same way, the number of MII oocytes needed to produce one blastocyst for clinical use was inferior in ICSI. This difference was always statistically significant except for the 38-45 years old female patients' group. This last result was in accordance with previous studies $[19,20]$ in which ICSI was shown to not improve reproductive outcomes for the couples with female patients over 38 years old [20] or over 39 [19]. From the present data, ICSI always has a higher efficiency compared to IVF due to a higher number of blastocysts available for direct transfer, freezing or other use (biopsy for preimplantation genetic testing). It was calculated that $26 \%(0.6 / 2.3)$ of surplus MII oocytes were needed to produce one blastocyst compared to ICSI. This value reached $38 \%$ in case of unexplained infertility and long ovarian stimulation protocol (respectively $(0.8 / 2.1$ and $0.7 / 1.8)$. Our results follow with Yovich et al. [17] that found a higher number of embryos to transfer in the ICSI group ( 2.5 versus 1.8 in IVF) in patients with mainly male factor issues. Mathematically, the highest number of clinically usable embryos in ICSI would increase the cumulative clinical rates.

The research of the best KPI to test ART efficiency and safety remains a subject of study. Different parameters are available to assess clinical management $[29,30]$ and laboratory performance and stability $[18,31]$. However, once the IVF laboratory has been monitored as a stable "tool" thanks to quality controls, it can be used to test procedures such as fertilization methods to produce a maximum of embryos for clinical use as done here. The comparison study of split insemination cycles from the same gamete cohorts (oocytes and spermatozoon) in the same ovarian cycle eliminates any clinical variants such as ovarian stimulation and responses that would be introduced in a non-sibling study [32-35]. The definition of the new KPI "number of MII oocytes to produce one clinically usable embryo" is online with Fisher and Scott that underlined the need for simple metrics to define fertilization success rates [34].

Previous authors applying split insemination reported data from embryo development until cleavage stage [10, $12,15,16]$ or on a minor number of cases $[11,13,14$, $18,28]$. The present study is the first one focused mainly on blastocyst stage, and on such a high number of cases 
that could also be analyzed according to different clinical parameters.

Because fewer embryos are produced in IVF compared to ICSI even with no male indication, conventional IVF and split insemination cycles should be carefully proposed based on the number of oocytes and the probability to produce embryos to transfer or to freeze. According to the number of MII oocytes available, the physician can calculate the expected number of embryos based on the laboratory data.

\section{Conclusion}

As per recent literature [36] and from the present study, clinical outcomes such as live-birth rates are comparable in ICSI and IVF in split insemination cycles. However, ICSI is more efficient in producing the highest number of blastocysts from the minimum number of MII oocytes compared to IVF in all patient groups and in particular for each ovarian stimulation protocol, infertility indication, or female age group. For the patients, the production of more embryos from one ovarian stimulation increases the chance of obtaining a pregnancy and a live birth. The long time effect on an increased cumulative live birth rate should confirm the present results. The novel KPI "number of MII oocytes to produce one clinically usable embryo" is an indicative parameter to test the clinical efficiency of ART procedures in the IVF lab.

\section{Authors' contributions}

SC and AG designed the study. SC wrote the manuscript and performed all data analyses. SC, CR, CA, GS, SR and RS performed IVF procedures. EM, AL, $C C$ and $A G$ enrolled patients and performed clinical investigations. $C R, A L, G S$, $\mathrm{SR}, \mathrm{RS}, \mathrm{EM}, \mathrm{AL}, \mathrm{CC}, \mathrm{MF}$ and $\mathrm{AG}$ contributed to manuscript writing. All authors approved the final manuscript.

\section{Funding}

No external funding was used for the study.

\section{Code availability}

Not applicable.

\section{Availability of data and materials}

All relevant data are within the text.

\section{Declarations}

Ethics approval and consent to participate

All procedures were ethically approved.

\section{Consent for publication}

All participants gave written consent to use of their data for research purposes and publication.

\section{Competing interests}

The authors have no competing interests.

\section{Author details}

${ }^{1}$ Unità di Medicina della Riproduzione - Centro HERA, via barriera del bosco, n. 51/53, Sant'Agata Li Battiati, Catania, Italy. ${ }^{2}$ Unit of Gynecology and Obstetrics-Department of General Surgery and Medical Surgical Specialties, University of Catania, Catania, Italy.

Received: 23 March 2021 Accepted: 16 July 2021

Published online: 26 July 2021

\section{References}

1. Steptoe PC, Edwards RG. Birth after reimplantation of a human embryo. Lancet. 1978;12;2(8085):366

2. Palermo G, Joris $H$, Devroey $P$, Van Steirteghem AC. Pregnancies after intracytiplasmic injection of single spermatozoa into an oocyte. Lancet. 1992;340:17-8.

3. Van Steirteghem AC, Nagy Z, Joris H, Liu J, Staessen C, Smitz J, Wisanto A, Devroey P. High fertilization and implantation rates after intracytoplasmic sperm injection. Hum Reprod. 1993;8:1061-6.

4. Hummel WP, Kettel M. Assisted reproductive technology: the state of the ART. Ann Med. 1997;29(3):207-14.

5. Benadiva CA, Nulsen J, Siano L, Jenning J, Givardis HB, Maier D. Intracytoplasmic sperm injection overcomes previous fertilization failure with conventional in vitro fertilization. Fertil Steril. 1999:72:1041-4.

6. Ludwig M, al-Hasani S, Küpker W, Bauer O, Diedrich K. A new indication for an intracytoplasmic sperm injection procedure outside the cases of severe male factor infertility. Eur J Obstet Gynecol Reprod Biol. 1997;75(2):207-10.

7. Gook DA, Edgar DH. Human oocyte cryopreservation. Hum Reprod Update. 2005;13:591-605.

8. De Geyter C, Calhaz-Jorge C, Kupka MS, Wyns C, Mocanu E, Motrenko T, Scaravelli G, Smeenk J, Vidakovic S, Goosens V. ART in Europe, 2014: results generated from European registries by ESHRE: The European IVFmonitoring Consortium (EIM) for the European Society of Human Reproduction and Embryology (ESHRE). Hum Reprod. 2018;33(9):1586-601.

9. Ministero della salute (2019) relazione del ministro della salute al parlamento sullo stato di attuazione della legge contenente norme in materia di procreazione medicalmente assistita 40/2004, 26 giugno

10. Plachot M, Belaisch-Allart J, Mayenga LM, Chouraqio A, Tesquier L, Serkine AM. Outcome of conventional IVF and ICSI on sibling oocytes in mild male factor infertility. Hum Reprod. 2002;17(2):362-9.

11. Van Landuyt L, De Vos A, Joris H, Verheyen G, Devroey P, Van Steirteghem A. Blastocyst formation in in vitro fertilization versus intracytoplasmic sperm cycles: influence of their fertilization procedure. Fertil Steril. 2005;83(5):1397-403.

12. Xie BG, Zhu WJ, Huang YH. Outcome of conventional IVF and ICSI on sibling oocytes in moderate oligoasthenozoospermia. Pakistan J Med Sci. 2013;29(5):1221-4.

13. Speyer B, O'Neill H, Saab W, Seshadri S, Cawood S, Heath C, Gaunt M, Serhal P. In assisted reproduction by IVF or ICSI, the rate at which embryos develop to the blastocyst stage is influenced by the fertilization method used: a split IVF/ICSI study. J Assisted Reprod Genetics. 2019;36:647-54.

14. Stimpfel M, Jancar N, Vrtacnik-Bokal E, Virant-Klun I. Conventional IVF improved blastocyst rate and quality compared to ICSI when used in patients with mild or moderate teratozoospermia. Syst Biol Reprod Med. 2019;65(6):458-64.

15. Yoeli R, Orvieto R, Ashkenazi J, Shelef M, Ben-Rafael Z, Bar-Hava I. Comparison of embryo quality between intracytoplasmic sperm injection and in vitro fertilization in sibling oocytes. J Assisted Reprod Genetics. 2008;25:23-8

16. Lee SH, Lee JH, Park YS, Yang KM, Lim CK. Comparison of clinical outcomes between in vitro fertilization (IVF) and intracytoplasmic sperm injection (ICSI) in IVF-ICSI split insemination cycles. Clin Exp Reprod Med. 2017:44(2):96-104.

17. Yovich JL, Conceicao JL, Marjanovich N, Ye Y, Hinchliffe PM, Dhaliwal SS, Keane KN. An ICSI rate of 90\% minimizes complete failed fertilization and provides satisfactory implantation rates without elevating fetal abnormatilies. Reprod Biol. 2018;18(3):301-11.

18. ESHRE Special Interest Group of Embryology and Alpha Scientists in Reproductive Medicine. The Vienna consensus: report of an expert meeting on the development of ART laboratory performance indicators. Reprod Biomed Online. 2017;35(5):494-510. 
19. Farhi J, Cohen K, Weissman A, Raziel A, Orvieto R. Should ICSI be implemented during IVF to all advantage-age patients with non-male factor subfertility? Reprod Biol Endocrinol. 2019;17(1):30-4.

20. Haas J, Miller TE, Nahum R, Aizer A, Kirshenbaum M, Zilberberg E, Lebovitz O, Orvieto R. The role of ICSI vs conventional IVF for patients with advanced maternal age - a randomized controlled trial. J Assisted Reprod Genetics. 38:95-100. Sterility. 2021;114(2):239-45.

21. Geng T, Cheng L, Ge C, Zhang Y. The effect of ICSI in infertility couples with non-male factor: a systematic review and meta-analysis. J Assisted Reprod Genetics. 2020;37:2929-45.

22. Bosch E, Espinos JJ, Fabregues F, Fontes J, Garcia-Velasco J, Llacer J, Requena A, Checa MA, Belliver on behalf of the Spanish Infertility SWOT Group (SISG). Always ICSI? A swot analysis. J Assisted Reprod Genetics. 37:2081-92.

23. Chamayou S, Patrizio P, Storaci G, Tomaselli V, Alecci C, Ragoia C, Crescenzo C, Guglielmino A. The use of morphokinetic parameters to select all embryos with full capacity to implant. J Assisted Reprod Genetics. 2013;30:703-10

24. Chamayou S, Alecci C, Ragolia C, Storaci G, Maglia E, Russo E, Guglielmino A. Comparison of in-vitro outcomes from cryopreserved oocytes and sibling fresh oocytes. Reprod BioMed Online. 2006;12(6):730-6.

25. Kuwayama M. Highly efficient vitrification for cryopreservation of human oocytes and embryos: the Cryotop method. Theriogenology. 2007:67:130-4.

26. Zegers-Hochshild F, Adamson GD, de Mouzon J, Ishihara O, Mansour R, Nygren K, Sullivan E, Vanderpoel S. International Committee for monitoring assisted reproductive rechnology (ICMART) and the world health organization (WHO) revised glossary of ART terminology, 2009. Fertil Steril. 2009;92(5):1520-4.

27. Franasiak JM, Forman EJ, Hong KH, Werner MD, Upham KM, Treff NR, Scott RT. The nature of aneuploidy with increasing age of the female partner: a review of 15,169 consecutive trophectoderm biopsies evaluated with comprehensive chromosomal screening. Fertil Steril. 2014;101(3):656-63.

28. Kim MS, Kim J, Youm HW, Park JY, Choi HY, Jee BC. Embryonic development in human oocytes fertilized by split insemination. Obstetrics Gynecol Sci. 2015;58(3):217-22.
29. Wilkinson J, Roberts SA, Vail A. Developments in IVF warrant the adoption of new performance indicators for ART clinics, but do not justify the abondonment of patient-centred measures. Hum Reprod. 2017;32(6):1155-9.

30. Pirtea P, de Ziegler D, Poulain M, Ayoubi JM. Which key performance indicators are optimal to assess clinical management of assisted reproduction cycles? Fertil Steril. 2020;114(1):24-30.

31. Hammond ER, Morbeck DE. Tracking quality: can embryology key performance indicators be used to identify clinically relevant shifts in pregnancy rate? Hum Reprod. 2019;34(1):37-43.

32. Tannus S, Son WY, Gilman A, Younes G, Shavit T, Dahan MH. The role of intracytoplasmic sperm injection in non-male factor infertility in advance maternal age. Hum Reprod. 2017;32(1):119-24.

33. Drakopoulos P, Garcia-Velasco J, Bosch E, Bloskeel C, de Vos M, SantosRibiero S, Makrigiannakis A, Tournaye H, Polyzos N. ICSI does not offer any benefit over conventional IVF across different ovarian response categories in non-male factor infertility: a European multicenter analysis. J Assisted Reprod Genetics. 2019;36(10):2067-76.

34. Fischer C, Scott RT. Three simple metrics to define in vitro fertilization success rates. Fertil Steril. 2020;114(1):6-8.

35. Sfontouris IA, Kolibianakis EM, Lainas GT, Navaratnarajah R, Tarlatzia BC, Lainas TG. Live birth rates using conventional in vitro fertilization compared to intracytoplasmic sperm injection in Bologna poor responders with a single oocyte retrieved. J Assisted Reprod Genetics. 2015;32:691-7.

36. Practice Committees of the American Society for Reproductive Medicine and the Society for Assisted Reproductive Technology. Intracytoplasmic sperm injection (ICSI) for non-male factor indications: a committee opinion. Fertil Steril. 2020;114(2):239-45.

\section{Publisher's Note}

Springer Nature remains neutral with regard to jurisdictional claims in published maps and institutional affiliations.
Ready to submit your research? Choose BMC and benefit from:

- fast, convenient online submission

- thorough peer review by experienced researchers in your field

- rapid publication on acceptance

- support for research data, including large and complex data types

- gold Open Access which fosters wider collaboration and increased citations

- maximum visibility for your research: over $100 \mathrm{M}$ website views per year

At BMC, research is always in progress.

Learn more biomedcentral.com/submissions 